\title{
Does virtual reality attract visitors? The mediating effect of presence on consumer response in virtual reality tourism advertising
}

\section{Wai Han Lo ${ }^{1}$ D $\cdot$ Ka Lun Benjamin Cheng $^{2}$}

Received: 28 April 2020 / Revised: 27 October 2020 / Accepted: 4 November 2020 /

Published online: 28 November 2020

(c) Springer-Verlag GmbH Germany, part of Springer Nature 2020

\begin{abstract}
An experimental study was conducted to test the mediating effect of presence on the consumer response to tourism promotion using virtual reality (VR) technology. Hong Kong college students $(N=203)$ were assigned to three experimental groups in which they were exposed to a hotel advertorial on an online blog, a VR $360^{\circ}$ video on a mobile phone, or a VR $360^{\circ}$ video using Google Cardboard goggles. The results supported the hypothesis that presence plays a mediating role in the relationship between the use of VR in tourism advertising and consumer response. As one of the first studies in the tourism field to investigate the effects of a virtual depiction of a real-world environment on consumption intentions, the findings reveal how VR technology can be used to promote a hotel by generating a sense of presence. This study makes a further contribution to the VR literature by finding that the use of Cardboard goggles did not produce a significantly greater sense of presence in the experience of advertorial viewers. The theoretical contributions and practical implications of the findings for the use of VR technology in branding and promotion are discussed.
\end{abstract}

Keywords Presence $\cdot$ Tourism $\cdot$ Advertising $\cdot$ Virtual reality $\cdot$ Cardboard

Wai Han Lo

janetlo@hkbu.edu.hk

Ka Lun Benjamin Cheng

bcheng@hkbu.edu.hk

1 Department of Journalism, Hong Kong Baptist University, Kowloon Tong, Hong Kong

2 School of Communication, College of International Education, Hong Kong Baptist University, Kowloon Tong, Hong Kong 


\section{Introduction}

Virtual reality (VR) has become an increasingly prominent tool for consumer marketing. Business analysts have suggested that the development of VR is comparable in importance with that of social media (Morris 2016), and that VR is likely to become a key medium for consumer marketing as it embarks on a new phase of development (Barnes 2016). Global communication firms have made huge investments in VR technologies in recent years. While VR technology has significant applications in many sectors, including healthcare, military, entertainment, education, and marketing, this paper focuses on the use of VR technology in the tourism field.

Virtual reality (VR) tools are of particular interest to tourism advertisers because they can provide potential consumers, wherever they are located, with a realistic preview of a travel experience. The interactivity, visualization, and immersion functions offered by VR technology can give consumers virtual experiences in simulated tourism environments to assist with their trip planning and decision making (William and Hobson 1995). As providing consumers with firstperson experiences of destinations helps to promote those destinations, various tourism organizations have begun to incorporate VR tools into their marketing and advertising strategies. For example, targeting newlyweds in New York, Marriott Hotels used VR to promote the honeymoon package offered at hotels in London and Hawaii. Potential consumers could take virtual tours that gave them an experience of staying at the hotels, visiting the cities' landmarks, and touring the surrounding areas. Qantas cabins are equipped with VR to allow passengers on selected flights to vicariously experience the Great Barrier Reef. Using Samsung Gear VR headsets, Thomas Cook takes consumers in Europe to Manhattan to enjoy a virtual helicopter ride that takes off from the balcony of the Santorini Hotel (Mandalbaum 2015).

Virtual reality (VR) become one of the most important and popular technologies nowadays (Barnes 2016). The increasing affordability of VR hardware means that more individuals now own VR equipment. A VR headset costs less than US\$100, and the Oculus Rift and Google Cardboard gear retail for around US\$500 and US\$7, respectively (Kabrovski 2017). This partly explains why $6 \%$ of the British population has its own VR hardware (YouGov 2017). International digital companies have made huge investments in VR technology. Facebook is one of the key players, having invested in 11 VR companies (Molla 2017). In 2017, Facebook introduced its first VR application, Facebook 360, allowing $360^{\circ}$ videos and photos to be posted. Google has also invested US\$300 million in VR start-up companies every year since 2016 (Shinta 2017). By 2025, VR-branded content is forecast to be worth US $\$ 80$ billion (Bellini 2016). VR is expected to become an important tool in advertising, especially in the travel industry (Kabrovski 2017). Although some research suggests that an audience that is more engaged and involved in VR advertisements are more likely to react positively towards the destination (Huang et al. 2012, 2016), there is a need to investigate how to optimize the use of VR as a tourism marketing tool (Yung and Khoo-Lattimore 2019). 
This study contributes to the literature by testing the mediating effects of a sense of presence on consumers' attitudes toward advertised products and their purchase intentions. It departs from most previous studies in three ways. First, while most VR research has focused on computer-generated, animated 3D environments, such as Second Life (Yung and Khoo-Lattimore 2019), this study is one of the first to investigate the effects of the virtual depiction of genuine (real-world) environments in the field of tourism. Tavakoli and Mura (2015) suggested that VR applications for tourism can make use of simulated 3D models of both real and imaginary places. In this paper, we examine the effect of VR simulations of real-world environments, and refer to this as genuine VR technology (Beck et al. 2019). Second, our study pioneers research into the use of paper goggles - a recent trend that has yet to be explored by scholars - for viewing $360^{\circ}$ VR tourism advertorials. Third, this is one of the first studies to test the transportation imagery model (TIM). Yung and KhooLattimore's (2019) systematic review of research into augmented reality (AR) and VR for tourism identified a lack of theoretical basis in the literature. The present study suggests that the TIM is a promising theoretical model for VR research.

\section{Literature review}

\subsection{The user experience of VR $360^{\circ}$ videos}

The French playwright Antonin Artaud coined the phrase "virtual reality" in the 1930s (Cogburn and Silcox 2014, p. 561). In this study, VR is defined as a computer-generated environment that allows a user to interact with various stimuli (Limniou et al. 2008) and experience scenery, objects, and sound effects in a virtual medium, giving the user the sense of actually "being there."

Most VR applications involve a computer-generated, 3D environment (Guttentag 2010), with some allowing individuals (represented by avatars) to interact with the virtual world in real time (Penfold 2009). One example is Second Life, in which avatars create and play in virtual spaces in real time (Huang et al. 2016). The metaanalysis of Yung and Khoo-Lattimore (2019) examined 46 peer-reviewed research articles on VR and AR published in the tourism field between 1996 and 2016 (see Table 1). Most of these studies (39\%) focused on Second Life virtual worlds, and investigated the effect of tourism marketing on Second Life (e.g., Mascho and Singh 2013; see Table 2). About 17\% addressed AR, which is defined as the enhancement of a real-world environment by layering computer-generated pictures (Guttentag 2010; Jung et al. 2015). From the meta-analysis, it can be seen that the study of genuine VR technology in promoting travel destinations is still in its infancy: only $11 \%$ of the studies addressed non-animated technology, including virtual meetings and virtual tutoring (Yung and Khoo-Lattimore 2019).

Over the past few years, more studies have examined the role of genuine VR 360 technology in the tourism field (see Table 3 ). The $360^{\circ} \mathrm{VR}$ video platform is a genuine VR technology that affords a high degree of immersion and a high level of visibility at a very reasonable cost, and is often used to deliver news content (Watson 2017). Wu and Lin (2018) examined the effects of $360^{\circ}$ video with different 


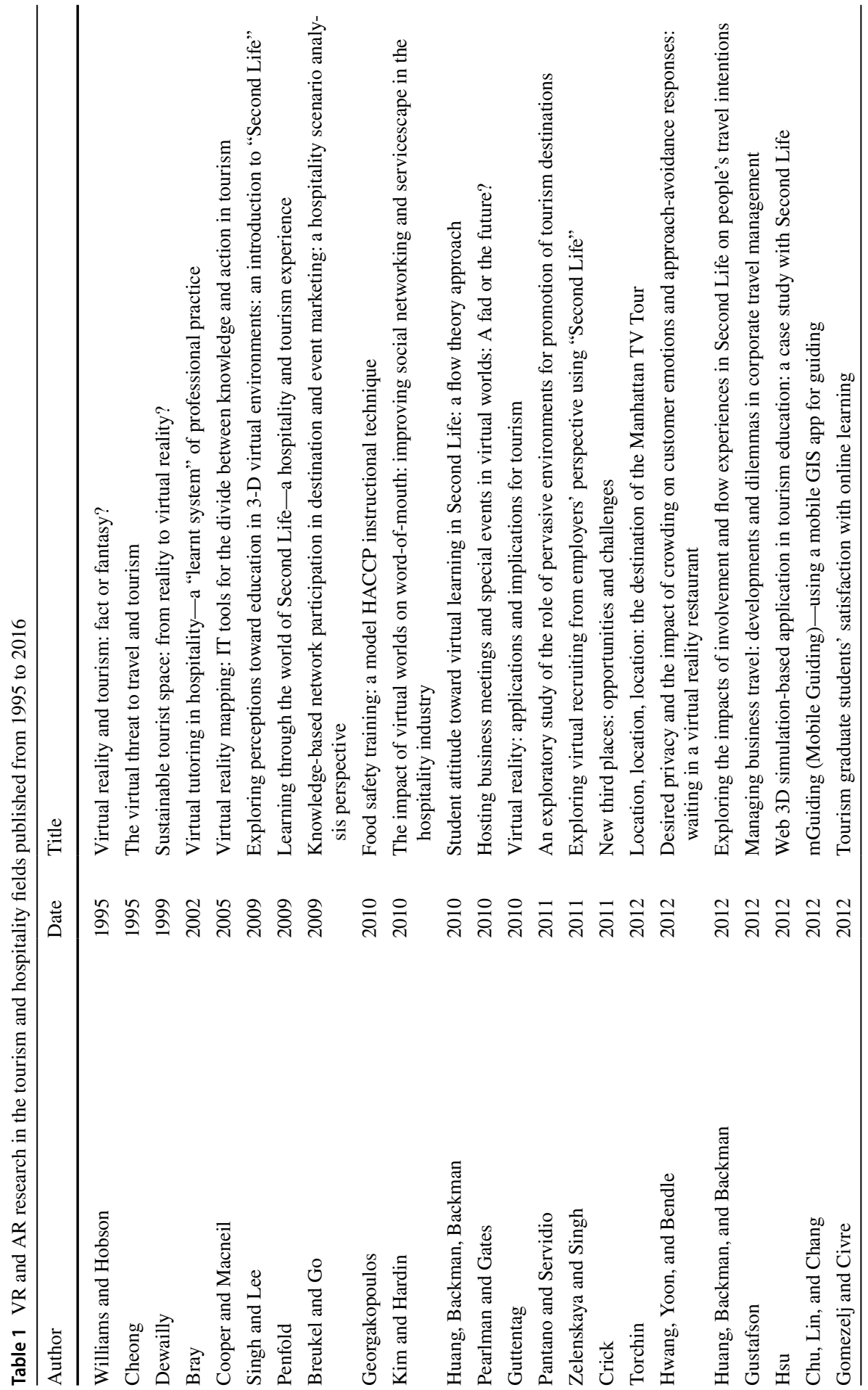




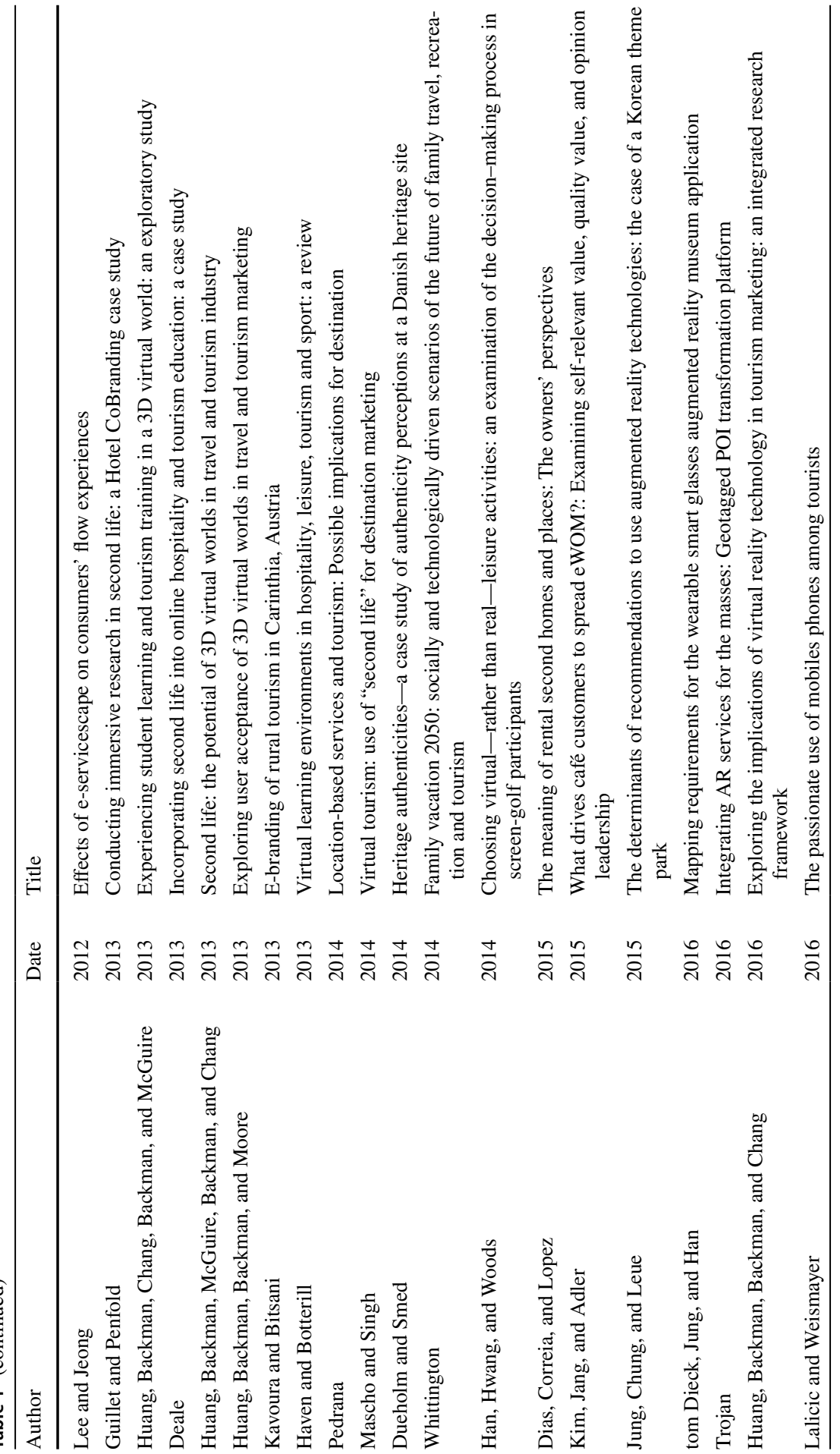




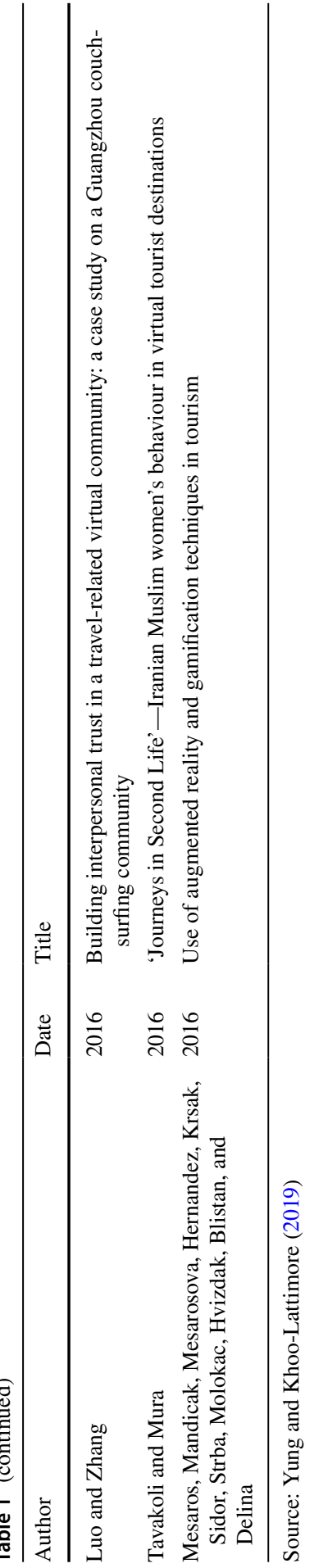


Table 2 Summary of the types of VR or AR covered in tourism and hospitality research published from 1995 to 2016

\begin{tabular}{lcl}
\hline Type of VR & No. of Studies & $\%$ \\
\hline Virtual Worlds & 18 & 39.13 \\
Virtual Environments & 11 & 23.91 \\
Augmented Reality & 8 & 17.39 \\
Virtual Reality & 6 & 13.04 \\
Virtual Communities & 3 & 6.52 \\
Total & 45 & 100 \\
\hline
\end{tabular}

Source: Yung and Khoo-Lattimore (2019)

perceptual systems, such as a head mounted display, tablet, and desktop computer. They found that the use of different perceptual systems had an impact on the effectiveness of an advertisement. Another study, by Wreford et al. (2019), found that current $360^{\circ}$ VR technology cannot replace live events, but it can encourage users to participate in future events (See Table 1). With previous studies mainly testing the effects of $360^{\circ} \mathrm{VR}$ video delivered through tablets and computers utilizing high-end devices, the present study is among the first to examine the effectiveness of genuine $360^{\circ}$ VR technology using mobile phones and cardboard viewers, while testing for the mediating role of presence.

For $360^{\circ}$ VR video, the real world is recorded in every direction using an omnidirectional camera. The audience can manipulate the directional orientation of the video by dragging the screen. The videos can then be viewed using smartphones, VR headsets, or Google Cardboard viewers, or on high-end devices (Lichterman 2017). High-end devices, such as head-mounted displays, sensors, or trackers, are the most popular for viewing $360^{\circ}$ videos, because they help to insulate users from the outside world (Simone et al. 2006) and thus enhance their experience of the virtual environment. However, these devices have several limitations; for example, they are often costly, heavy, and uncomfortable to wear (Sharples et al. 2008). Alternatively, a low-cost $360^{\circ} \mathrm{VR}$ experience is available to any user with a smartphone and a Cardboard device. Cardboard users are insulated from the outside world by having their eyes covered. They can watch VR $360^{\circ}$ videos in every direction, although they are not able to interact with the environment that is portrayed (The Guardian 2017). This technology places the audience at the center of the scene.

\subsection{Presence}

It is important to examine the experience of presence in virtual environments because this has been found to influence the impact of the overall VR experience (Villani et al. 2007) and the degree to which audiences are transported into a VR environment (e.g., Fox et al. 2009). Presence was conceptualized by Lombard and Ditton (1997) in six dimensions. The first dimension is immersion, referring to perceptual and psychological involvement. The second dimension is realism, which is either perceptual or social. The third dimension is transportation, or the audience's sense of being in the virtual world. The fourth dimension is parasocial interaction. 


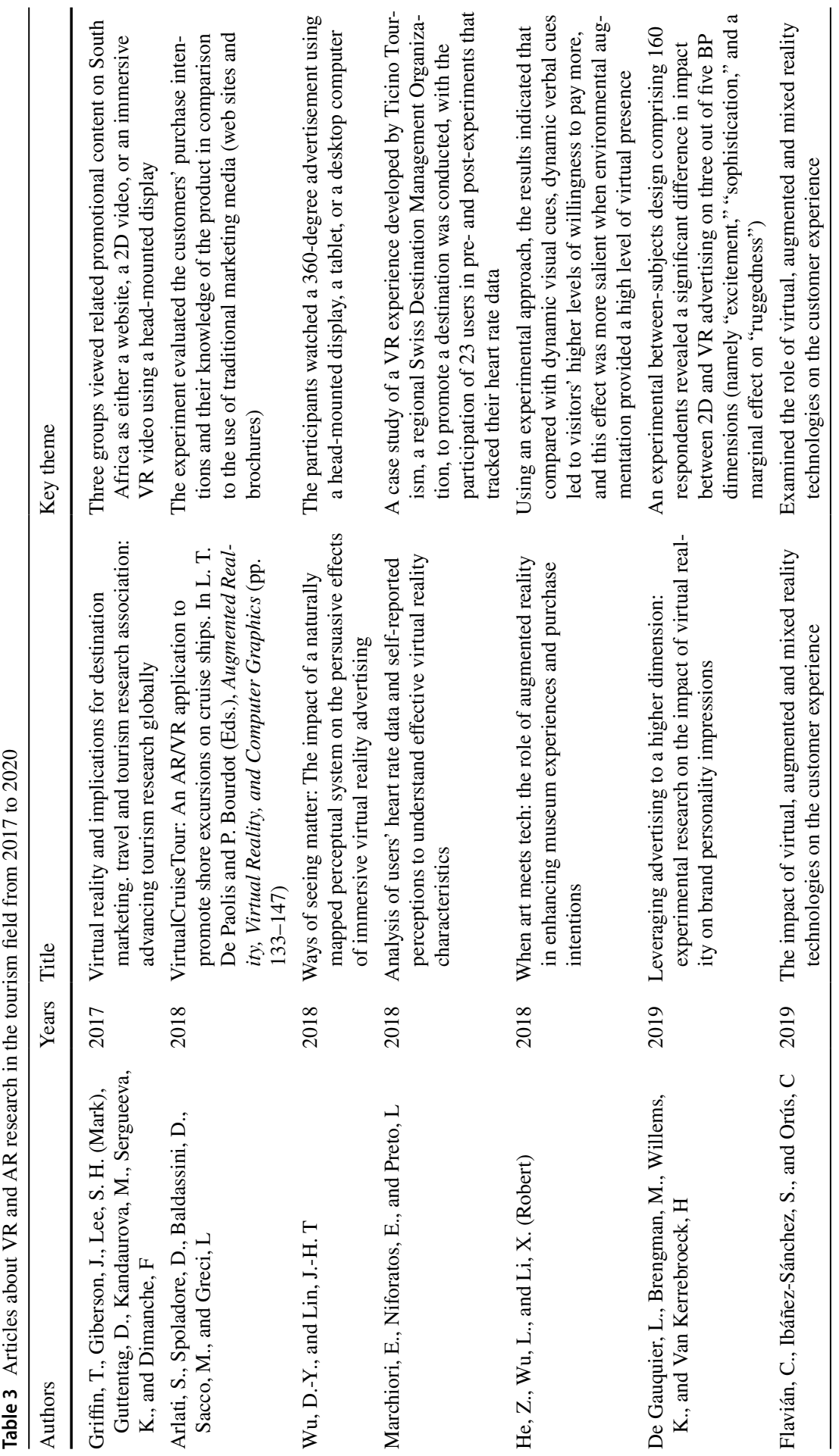




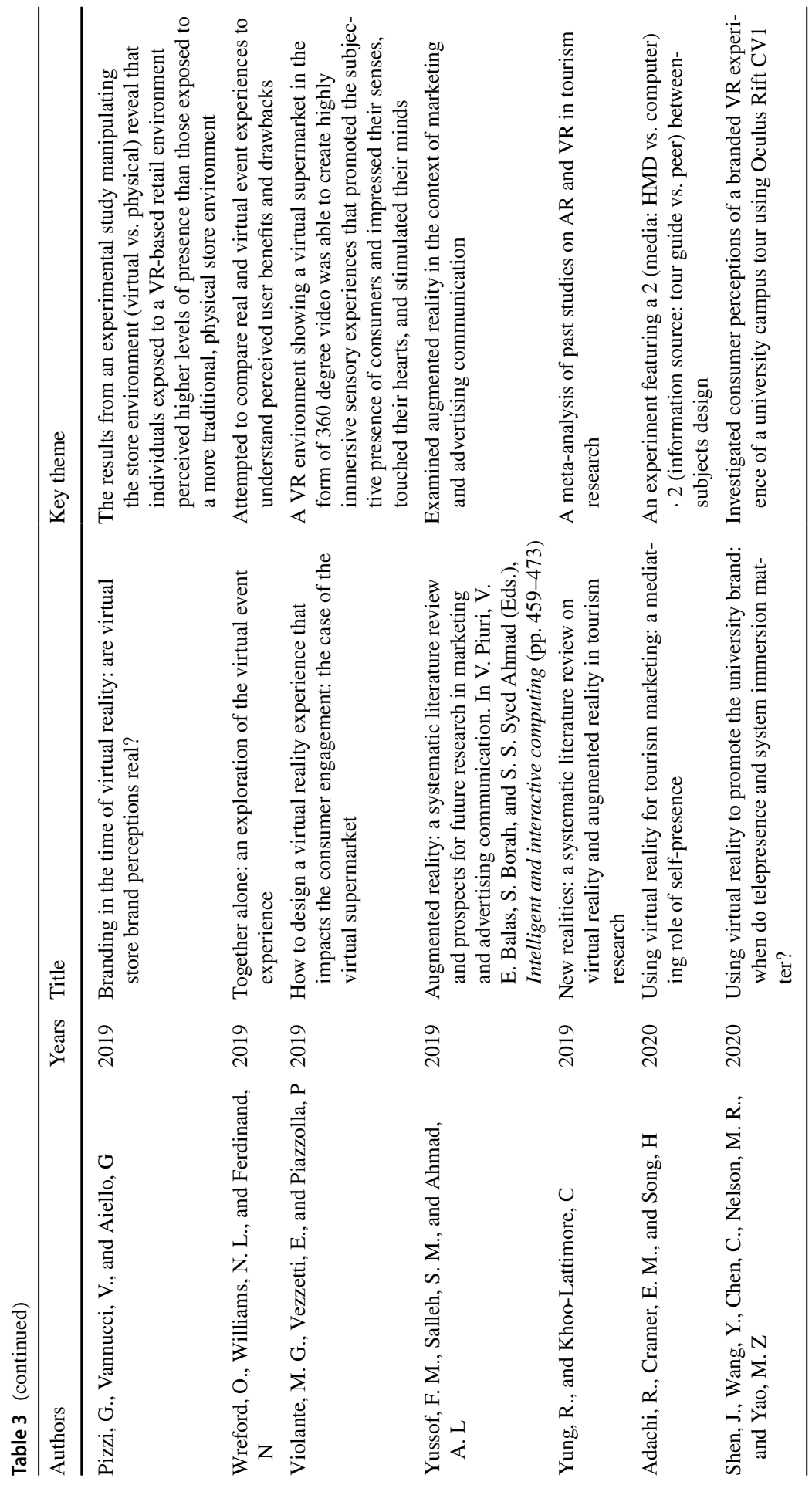


The fifth dimension is the perception of the medium as a social actor. The last dimension is social richness, or the degree to which the audience perceives the medium as warm and sociable (Lombard and Ditton 1997).

All of these dimensions can be captured in a single definition, namely the feeling of being lost in a medium (Bracken 2005) or of being in a mediated world (Steuer 1992) when one is not physically in the environment depicted. Presence can also refer to the mental process by which an audience perceives a mediated environment as non-mediated and experiences virtual objects as actual objects (Lee 2004). Lombard and Ditton (1997) defined presence as "the perceptual illusion of nonmediation." In other words, it is a psychological state in which audience members forget their surrounding environment and gain a sense of being immersed in the mediated world, allowing them to perceive remote people as nearby (Bracken 2005). The audience experiences this illusion via cognitive, affective, and human sensory processing systems (Lombard et al. 2000).

Presence is now an important concept in various disciplines, such as communication, psychology, and computer science. The concept of presence improves the understanding of audiences' experiences of television, online news, virtual environments, and video games (Kim and Biocca 1997; Lombard and Ditton 1997; Cheng and Lo 2017). However, little empirical research has been conducted on the concept of presence (Bracken 2005; Cheng and Lo 2015). Debbabi and Baile (2005) also pointed out that few researchers have examined the factors that induce a sense of presence, although the characteristics of the medium represent one potential factor. For example, studies have found that higher resolution images are more effective in eliciting and experience of presence from an audience (e.g., Neuman 1990; Grabe et al. 1999), and that the use of animation in news reports may intensify the experience of presence (Cheng and Lo 2015; Lo and Cheng 2015). Hamman (2006) suggested that a sense of presence could be enhanced by personalized media content.

Scholars have explored the experience of presence in various fields, such as education, health care, entertainment, and news. For example, experiencing news content through VR may affect the audience's understanding of news stories because of a feeling that they are present in the actual place, alongside the news reporter (Jon 2016). In the tourism field, a study by Gutierrez et al. (2008) suggested that tourists were physically and psychologically immersed in VR technology. Furthermore, Sylaiou et al. (2010) found that the degree of presence and sense of enjoyment were positively related. In this study, we examine presence as a potential mediator in the effects of using VR technology in advertorials on audiences' attitudes toward the advertised products and their purchase intentions.

\subsection{The experience of presence in VR $360^{\circ}$ videos}

Witmer et al. (2005) argued that four factors influence the experience of presence: control, sensory dimensions, distraction, and realism. Control refers to the audience's capacity to act on the virtual world. Sensory dimensions determine the representational richness of the media channels. This in turn affects the vividness of the medium, which is the most important determinant of presence (Steuer 1992). A 
more vivid mediated environment is one with a higher resolution and more sensory dimensions in each of its perceptual channels (Fortin and Dholakia 2005; Steuer 1992). Distraction refers to the degree to which the audience is distracted by external or internal factors. Realism has two sub-dimensions: (1) the degree to which the virtual environment is similar to the real world, and (2) the meaningfulness of the audience's experience.

Interactivity, audio and visual features, and the content of the virtual world all contribute to the experience of presence (Lombard and Ditton 1997). Users have more control over, and a greater ability to interact with, a video medium when using VR $360^{\circ}$ video than when viewing a blog, because the former allows them to explore the surrounding sights and sounds by changing direction, either by dragging the screen or through head movements. Furthermore, $360^{\circ}$ video has more sensory input and output than normal video. Studies of news reports have found that higher resolution images and background music increase the sensory breadth and depth of VR content (Reeves and Nass 1996; Rizzo et al. 1998). The capacity for movement and touch contribute to the vividness of VR $360^{\circ}$ video (Park et al. 2008). By increasing vividness, the use of VR $360^{\circ}$ cameras helps to transport users into the VR world, giving them a greater sense of being in the environment portrayed.

The audience experiences an even greater sensation of realism when viewing VR $360^{\circ}$ videos using Cardboard. Viewers using Cardboard devices to watch VR $360^{\circ}$ videos may be less distracted by their real physical environment than those watching via browsers or non-immersive screens, as Cardboard users have their eyes covered and are thus insulated from the physical environment (Biocca and Delaney 1995). Being forced to concentrate on the video creates a greater sense of physical immersion in the scenes portrayed. The use of a VR $360^{\circ}$ camera thus provides an immersive experience that may make the mediated environment feel more realistic and meaningful. Chessa et al. (2016) found that high-end wearable devices can evoke a higher level of presence, due to the users' insulation from the outside world. The audience can watch VR $360^{\circ}$ videos in every direction, which places them at the center of the scene portrayed and makes them feel as if they are experiencing a real place alongside the other people depicted at the scene.

Based on this theoretical discussion and the findings of past studies, we propose the following hypothesis.

H1: Viewers experience different levels of intensity of presence when viewing advertorials using different media: a VR $360^{\circ}$ video viewed through Google Cardboard goggles offers the most intense sense of presence, followed by a VR $360^{\circ}$ video viewed on a mobile phone, with a non-VR blog post offering the least sense of presence.

\subsection{Attitude toward the product}

Individuals' attitudes toward a product reflect their judgment and assessment of the product. Individual judgments of the product's attributes and the effectiveness of the product's advertising both contribute to an audience's overall attitude toward the product (Mitchell and Olson 1981). As Giner-Sorolla (1999) suggested, attitude 
comprises three components. First, attitude relates to its object-in our case, the advertised hotel. Second, attitude is evaluative, which means that it is based on an assessment of the degree to which the object is good or bad. Third, attitude is the result of internal assessment; as a cognitive response, it differs from the feelings elicited by a product.

Product images have been found to be associated with the experience of presence and positive emotions (Drenger et al. 2008). Studies have shown that the characteristics of a medium may enhance the experience of presence and in turn influence brand perceptions, especially attitudes toward the branded product (Klein 2003). In other words, presence mediates the relationship between the formal characteristics of a medium, brand perceptions, and the branded products. As Lombard and Ditton (1997) argued, audience members gain a sense of involvement and engagement when they experience presence. This makes the message more persuasive and thus more readily accepted (Kim and Biocca 1997). Education scholars have argued that users learn better and faster and are more easily persuaded if they are highly immersed in a learning activity (Huang 2003; Reid 2004). A greater sense of presence also increases users' loyalty to websites (Cyr et al. 2007). Strange and Leung (1999) suggested that individuals are more likely to accept the messages conveyed by a narrative if they are immersed in the narrative and experience a sense of presence. In a study of the persuasiveness of advertisements, Grigorovici (2003) reported that viewers found advertising messages more persuasive when they experienced a higher level of presence. Two further studies have suggested that the experience of presence in virtual tours of cities led to a change in viewers' attitudes towards the destination and their level of enjoyment (Tussyadiah et al. 2017, 2018). Thus, we hypothesize that presence has a positive effect on consumers' attitudes toward a product, which is represented in this study as an advertised hotel.

H2: A sense of presence while viewing an advertisement has a positive effect on consumers' attitudes toward a hotel featured in the advertisement.

\subsection{Purchase intention}

Purchase intention is an important variable that can be used to measure the behavioral motivation elicited by an advertisement. Whereas attitude is evaluative, purchase intention is regarded as an individual's behavioral tendency (Bagozzi et al. 1979) and reflects the motivation to consciously plan to carry out a certain action (Eagly and Chaiken 1993). Thus, purchase intention can be defined as "an individual's conscious plan to make an effort to purchase" (Spear and Singh 2004, p. 56). It is a commonly used and effective measure of a consumer's likelihood of buying a product and the probable behavior of a consumer in response to an advertisement (Andrews et al. 1992; Beerli and Santana 1999; Li et al. 2002).

Fishbein and Ajzen (1975) asserted that attitude influences behavioral intention, which in turn affects behavior. Brand attitude is positively related to the intention to commit to a brand and to the associated product and organization. Research has shown that individuals who hold a positive image of an organization have a greater intention to seek employment at that organization via its recruitment website (Allen 
et al. 2007). A positive product attitude also increases viewers' loyalty (McDowell and Sutherland 2000) and changes individuals' behavior (Morris et al. 2002). Thus, we propose the following hypothesis.

H3: More positive attitude toward a hotel featured in an advertisement has a positive impact on purchase intention.

\subsection{The transportation imagery model (TIM)}

In sum, this study examines the impact of VR advertorial content on users' experience of presence, and tests whether this experience influences attitudes toward the product, and whether attitudes in turn affect purchase intentions. The TIM can explain the relationship between these variables, and has been used extensively in studies of presence because it is based on similar assumptions (Bracken 2005). Presence can also be conceptualized as a dimension of transportation. Both transportation and presence describe the audience's sense of "being there" (Lombard and Ditton 1997).

The TIM assumes that a medium with a high degree of interactivity and vividness is better able to transport the audience out of the real world and into the narrated world. For example, audience members' reactions to news coverage is influenced by their transportation into the virtual environment of a narrated world (Green and Brock 2000), and they may even suspend their existing views. The TIM can therefore be used as a framework for analyzing individuals' reactions to news stories (Bracken 2005). Extending this to tourism research, the TIM explains how $360^{\circ}$ video and the use of VR goggles give viewers the sense of experiencing travel destinations first hand.

This study proposes that the use of VR technology affects viewers' assessment of advertorials by enhancing their sense of presence. We expect VR technology to "transport" the audience into the world depicted on video and intensify their experience of presence. It is further proposed that this may positively affect users' attitudes toward the product, which may in turn have an impact on their purchase intentions. Specifically, this paper suggests that compared with blog readers, viewers who use immersive media with VR technology will have a more intense experience of presence, and thus will hold more positive attitudes toward an advertised hotel and have higher intentions to make reservations at the hotel. The proposed path model is shown in Fig. 5. The hypothesis based on the TIM is as follows:

H4: The use of VR technology affects viewers' assessment of advertorials by

transporting viewers out of the real world and into the narrated world.

\section{Methodology}

An experiment was conducted with 203 college students majoring in communication, recruited from a medium-sized University in Hong Kong. The mean age of the participants was 20.4. Male participants made up 32\% of the sample $(n=65)$ and female participants $68 \%(n=138)$. Seventy-six of the participants were in the second 


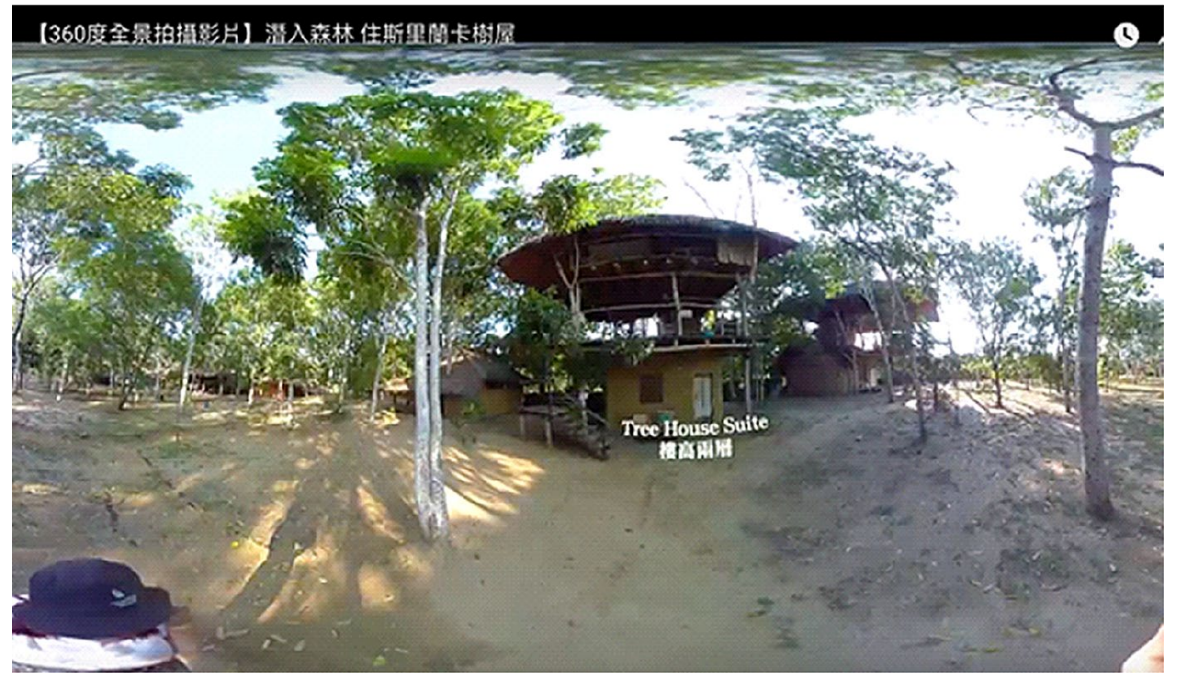

Fig. 1 Sample VR $360^{\circ}$ video frame showing the hotel

year of their course, 68 were in their third year, and 59 were in their fourth. The participants were randomly assigned to one of three experimental conditions: the blog group (Condition 1) read a blog post, the VR $360^{\circ}$ group (Condition 2) viewed a VR $360^{\circ}$ video on a mobile phone, and the Cardboard group (Condition 3 ) a VR $360^{\circ}$ video on a mobile phone wearing Google Cardboard goggles. The participants also completed a pre-test and post-test pen-and-paper questionnaire.

\subsection{Stimuli}

A VR $360^{\circ}$ advertorial video from the travel magazine Eat and Travel Weekly was chosen as the stimulus for Conditions 2 and $3^{1}$ (see Figs. 1 and 2 for sample frames). Eat and Travel Weekly, published by Next Media, specializes in food and travel and is one of the most popular travel publications in Hong Kong. As the magazine no longer has a print run, its content is now produced solely for websites and mobile applications.

The chosen VR $360^{\circ}$ advertorial video featured a treehouse hotel in Sri Lanka and the surrounding area and facilities. The footage allowed the audience to join the reporter as she visited a suite in the treehouse hotel, breakfasted next to a nearby lake, experienced the hotel's facilities, and toured the surrounding environment. The video first showed the first and second floors of one of the hotel's suites, as the reporter walked through a sitting room and bedroom. It then featured the facilities of the hotel, such as its swimming pool and restaurant. The reporter then left the hotel to take breakfast with a friend next to a nearby lake. The VR $360^{\circ}$ advertorial video

\footnotetext{
1 The $360^{\circ}$ video stimulus is available at https://www.youtube.com/watch? $\mathrm{v}=\mathrm{AbLwa} 1 \mathrm{JUqMc}$.
} 


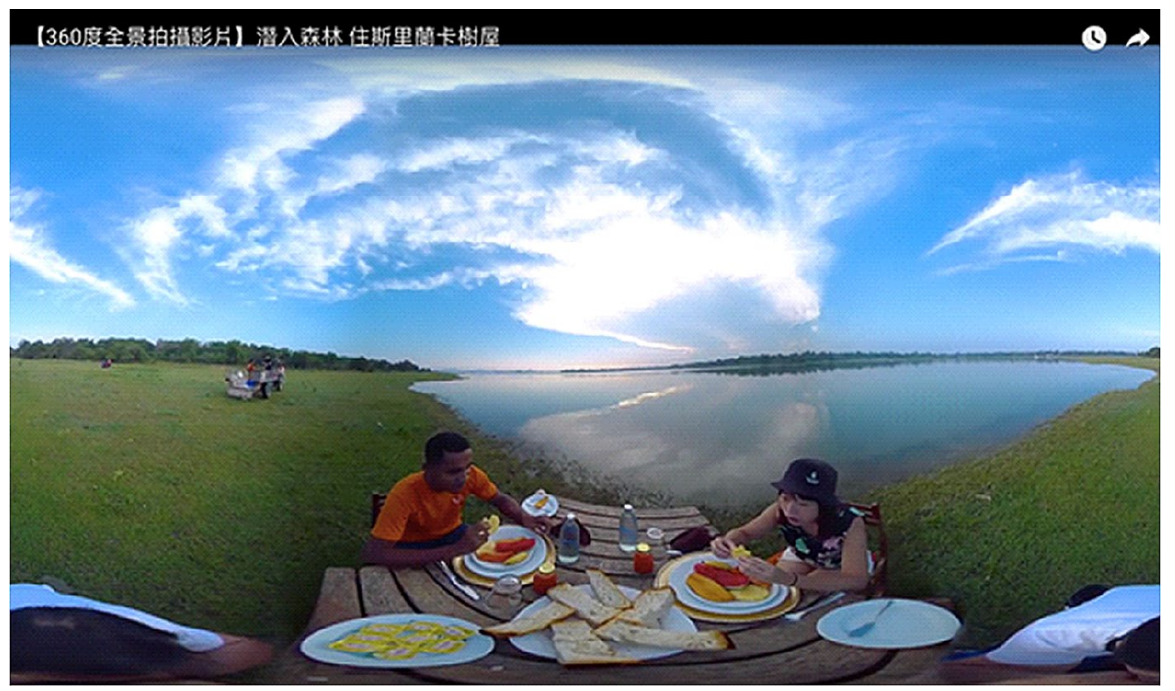

Fig. 2 Sample VR $360^{\circ}$ video frame showing the lake

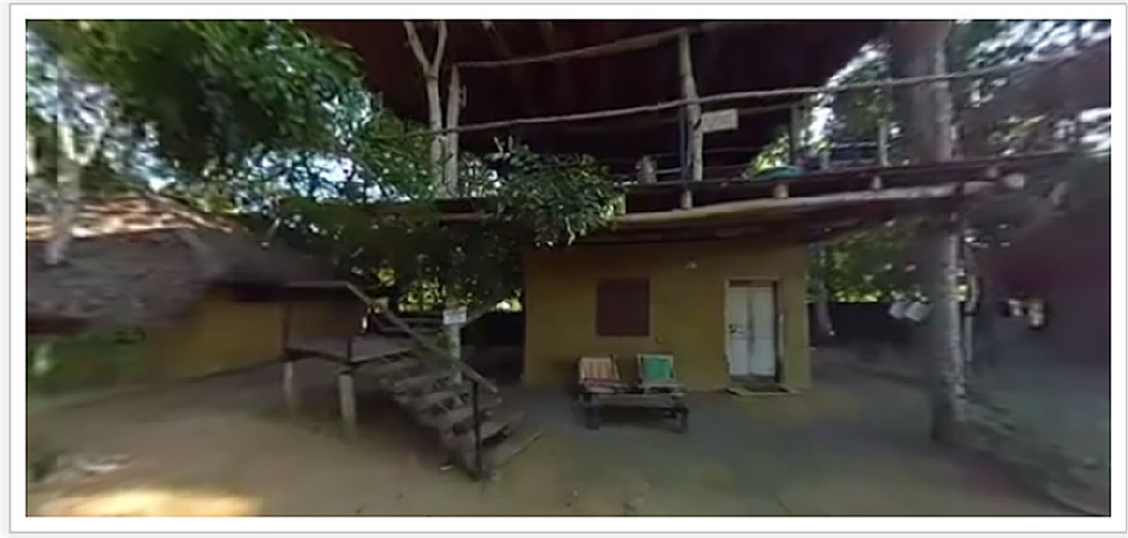

Tree House Suite, 樓高兩層

Fig. 3 Sample blog post image showing the hotel

lasted for $1 \mathrm{~min}$ and $34 \mathrm{~s}$. Chinese subtitles were used to describe the details of each location. The Eat and Travel Weekly logo was shown at the end of the video.

The manipulated version of this stimulus (used in Condition $1^{2}$; see Figs. 3 and 4 for sample images) was an edited blog post on the same hotel. To ensure that similar

\footnotetext{
${ }^{2}$ The manipulated blog post is available at http://o365.hkbu.edu.hk.
} 


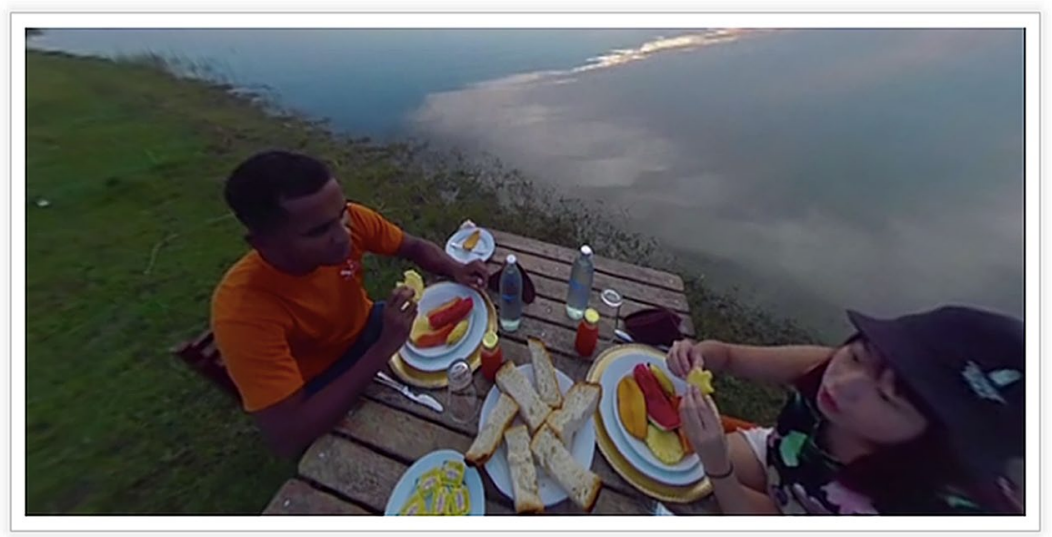

早上起來時, 更可到附近湖泊旁享用早餐, 好風光, 好寫意。

Fig. 4 Sample blog post image showing the lake

information was provided by the video and on the blog post, all of the photographs used in the blog post were captured from the video footage, with the first shot of each location included in the blog post. The subtitles appended to the video footage were used as the text of the blog post. This manipulated version of the original stimulus resembled the original video produced by the travel website, but in a blog post format. The logo of East and Travel Weekly was also shown on the blog post.

Ten college students were recruited for a screening test to ensure that the quality of the blog post was equivalent to that of conventional travel blogs. The blog post was further edited to address the comments collected from the screening test. The VR $360^{\circ}$ video and the blog post were then assessed to ensure they were of the same quality, and both were found to be comparable to the actual travel content produced by the media organization.

\subsection{Measurement instrument}

The experiment was conducted to investigate the effects of using VR in tourism advertising on viewers' experience of presence, attitudes toward an advertised hotel, and purchase intentions (see Table 4). The measurement instrument was a questionnaire measuring these three variables and some additional control variables.

\subsection{Presence}

The participants were asked to rate five statements, such as "The viewing experience was involving" and "The viewing experience was intense," on a 6-point scale $(1=$ strongly disagree, $6=$ strongly agree $)(\alpha=0.92)$. This measure was modeled on the scale proposed by Nowak and Biocca (2003). 
Table 4 Measurement instruments for multi-item variables

\begin{tabular}{ll}
\hline Variable & Item \\
\hline Presence & The experience of viewing the video was involving \\
& The experience of viewing the video was intense \\
& I felt like I was inside the environment I saw in the video \\
& I felt surrounded by the environment I saw in the video \\
& I felt immersed in the environment I saw in the video \\
& The hotel is good \\
& The hotel is of a high quality \\
Positive attitude towards the hotel & Do you like the hotel? \\
& I would consider booking this hotel \\
Purchase intention & It is highly probable that I will book this hotel \\
& I am willing to book this hotel \\
\hline
\end{tabular}

\subsection{Attitude toward the advertised hotel}

The participants were asked to rate the advertised hotel using a 3-item, 6-point semantic differential scale, with 1 indicating a more negative evaluation and 6 indicating a more positive evaluation (bad/good; low quality/high quality; dislike/like) $(\alpha=0.91)$. This measure was adapted from the scale developed by Sääksjärvia and Samiee (2011).

\subsection{Purchase intention}

To measure purchase intention, three items were rated on a 6-point scale $(1=$ strongly disagree, $6=$ strongly agree $)(\alpha=0.95)$. The items included the statement "If I were to travel to Sri Lanka and budget allowed, I would consider booking this hotel." This scale was adapted from Ali (2016).

\subsection{Control variables}

Consumers' motivation to visit a destination is likely to influence their evaluation of an advertisement promoting the destination and related tourist spots (Yoon and Uysal 2005). As a control variable, prior motivation was measured by asking the participants to rate their likelihood of traveling to Sri Lanka if time and budget allowed, using a 6 -point scale $(1=$ not likely; $6=$ very likely). To minimize the prompt effect, the participants were also asked to rate six other locations. The list of locations was compiled by randomly selecting from the top 10 travel destinations most frequently searched on Google by Hong Kong people in 2016, as identified in a study conducted by a well-established Hong Kong travel organization (Skyscanner 2017). These included both short-distance (e.g., Japan and Singapore) and long-distance (e.g., the U.K. and Canada) travel destinations. The order of the seven 
destinations, including the target destination, was randomized. The participants were asked to rate these destinations before being exposed to the stimuli.

As the VR $360^{\circ}$ video selected as the stimulus had been released to the public, the participants could have been exposed to the video prior to the experiment. Such prior exposure to the video could have influenced their evaluation of the advertorial and the hotel advertised, as repeated exposure to media content influences perceptions of that content (e.g., Moorthy and Hawkins 2005; Zaragoza and Mitchell 1996). In Conditions 2 (the VR $360^{\circ}$ group) and 3 (the VR Cardboard group), the participants were asked to indicate whether they had watched the VR $360^{\circ}$ video before the experiment, and the number of times they had watched it.

In all of the conditions, due to resource constraints, the participants were asked to use their own mobile phones to view the stimuli. The screens of their mobile phones were differently sized, which might have influenced their experience of presence (e.g., Hou et al. 2012). In the last part of the questionnaire, therefore, they were asked to indicate the brands and models of the mobile phones they were using. Although the literature has shown that the resolution, and resulting clarity, offered by a viewing device may influence viewers' experience of presence (Bracken 2005), resolution was not included as a control variable, as it was impossible to compare the screen resolution of different mobile phone brands in a unified way.

\subsection{Procedure}

The participants were first briefed to provide them with necessary information on the study. They were then asked to complete the first part of the questionnaire, which measured prior motivation to visit the target destination as a control variable. Next, the participants were randomly assigned to one of the three conditions, exposing each participant to one of three stimuli. In Condition 1, the participants were given a link to an online blog and asked to read the blog post using their own mobile phones for as long as they wanted. In Condition 2 (the VR $360^{\circ}$ group), the participants were given a link to a VR $360^{\circ}$ video and asked to view the video once on their own mobile phones. In Condition 3 (the VR Cardboard group), each of the participants was given a pair of Cardboard goggles and asked to insert his/her mobile phone into the device before viewing the VR $360^{\circ}$ once through the goggles. Before viewing the stimulus, a 3-min training session using a VR $360^{\circ}$ video with entertainment content irrelevant to the aim of the study was conducted to familiarize the participants in Condition 3 with the use of the Cardboard goggles. As this technology is still new to young people in Hong Kong, it was important to ensure that the participants had mastered the technique of viewing VR $360^{\circ}$ video with Cardboards before being exposed to the stimulus, to confirm that they were actually able to view the VR effects created by the stimulus. All of the participants in Condition 3 reported that they had mastered the technique during the training session. A link to the VR $360^{\circ}$ video stimulus was then given to the participants, who were asked to use their Cardboards to view the video once.

In all three conditions, the participants were asked to complete the second part of the questionnaire, which measured the variables of major interest, after exposure to 
the stimuli. In the last part of the questionnaire, the participants in Conditions 2 and 3 were asked to indicate whether they had watched the video before the experiment, and the number of times they had watched it if so. All of the participants were asked to provide the brands and models of their mobile phones. Demographic data, such as gender, age, and year of study, were also solicited in this part of the questionnaire. At the end of the experiment, the participants were debriefed, thanked, and dismissed.

\section{Results}

Two hundred and three participants took part in the experiment, and all of the cases were considered valid. Seventy-eight participants were assigned to Condition 1 (the blog group), 63 to Condition 2 (the VR $360^{\circ}$ group), and 62 to Condition 3 (the VR Cardboard group). An index was created by averaging the scores for the multiitem scales. Mean scores were calculated for the variables of interest, namely presence $(M=3.42)$, attitude toward the advertised hotel $(M=3.72)$, and purchase intention $(M=3.5)$. The linear regression results showed that age and year of study had no effect on any of these variables $(p>0.05)$. The results of an independent samples t-test revealed that gender also had no significant influence on the variables $(p>0.05)$.

\subsection{Control variables}

Regarding the control variables, the linear regression results indicated that prior motivation had a significant positive effect on attitude toward the advertised hotel $(\beta=0.25, p<0.001)$ and on purchase intention $(\beta=0.14, p=0.05)$, but not on presence $(\beta=0.10, p=0.14)$. This variable was controlled for in the subsequent tests. In regard to prior exposure, only six of the participants in the VR $360^{\circ}$ and VR Cardboard groups $(4.8 \%)$ reported that they had watched the video before the experiment. The mean frequency of prior exposure was 1.5. As this value was not statistically meaningful, this control variable was not included in the subsequent tests. To control for screen size, the reported brands and models of the participants' mobile phones were coded into size measured in inches. The mean screen size was 4.88 inches. Screen size was found not to have a significant effect on presence $(\beta=0.02$, $p=0.82$ ). Therefore, it was not included in the subsequent tests.

\subsection{Hypothesis testing}

H1 proposed that viewers would experience different levels of intensity of presence when viewing advertorial content using different immersive media: VR $360^{\circ}$ video viewed via Cardboard goggles would offer the most intense sense of presence; a VR $360^{\circ}$ video viewed on a mobile phone would offers the second most intense sense of presence; and a blog post would offer the least intense sense of presence. The results from a one-way ANOVA revealed significant differences between the three 


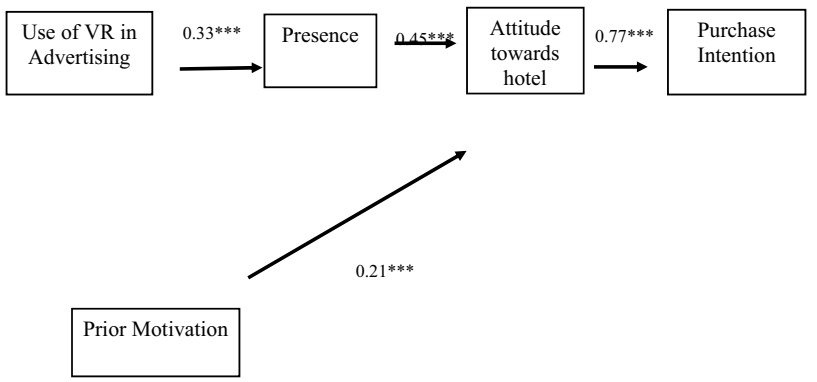

Note 1: Model fit indexes: SRMR $=.08 ; \mathrm{NFI}=.90$

Note 2: For the use of VR in advertising, viewing the blog post was coded as 0; using VR was coded as 1 .

Note $3: * p<.05, * * p<.01, * * * p<.001$

Fig. 5 Path model of the effects of using immersive media in advertising

groups in terms of presence $\left(F=11,{ }^{2}=0.10, p<0.001\right)$. A post-hoc Tukey's HSD test showed that the blog group reported experiencing a significantly lower intensity of presence $(M=2.99, S D=0.90)$ than their counterparts in the other two groups. The VR $360^{\circ}$ group $(M=3.75, S D=1.06)$ scored slightly higher than the VR Cardboard group $(M=3.63, S D=1.13)$ for presence, but this difference was not significant. Therefore, $\mathrm{H} 1$ was partially supported.

$\mathrm{H} 2$ proposed that presence would have a positive effect on attitude toward the advertised hotel. The linear regression results show that presence had a significant positive effect on attitude toward the advertised hotel after controlling for prior motivation $(\beta=0.43, p<0.001)$. Thus, $\mathrm{H} 2$ was supported.

$\mathrm{H} 3$ proposed that attitude toward the advertised hotel would have a positive effect on purchase intention. The linear regression results show that attitude toward the advertised hotel had a significant positive effect on purchase intention after controlling for prior motivation $(\beta=0.75, p<0.001)$. Therefore, $\mathrm{H} 3$ was supported.

We proposed a path model to determine the role of presence in mediating the relationship between the use of VR in advertising and viewers' attitudes and purchase intention. To test the proposed model, PLS-SEM analysis was adopted (see Fig. 5). As there was no significant difference in presence between the VR $360^{\circ}$ group and the VR Cardboard group, while the blog group reported experiencing a significantly lower level of presence, the VR $360^{\circ}$ and the VR Cardboard groups were combined, and a dummy variable was created for the analysis $(0=\mathrm{blog}, n=78$; $1=\mathrm{VR}$ group, $n=125)$. As prior motivation was found to influence attitudes toward the advertised hotel and purchase intention, this variable was entered into and controlled for in the model. The factor analysis result shows that all relevant items were loaded onto the designated latent variables (factor loading $\geq 0.80$ ) and there were no cross-loadings to other latent variables.

All of the proposed paths in the model were found to have significantly positive relationships (see Fig. 5). The paths between the use of VR in advertising and presence $(\beta=0.32, p=0.000)$; presence and attitude toward advertised hotel $(\beta=0.45$, $p<0.001)$; and attitude toward advertised hotel and purchase intention $(\beta=0.77$, 
$p<0.001)$ showed moderate-to-large direct effect sizes. The indirect paths between the use of VR in advertising and attitude toward advertised hotel $(0.15)$ and purchase intention (0.11) showed moderate mediating effect sizes. The indexes of SRMR $(0.08)$ and NFI (0.90) showed a good model fit. Therefore, H4 was supported.

\section{Discussion}

The participants experienced a more intense sense of presence when viewing the advertorials in the form of a VR $360^{\circ}$ video, with or without Cardboard goggles, than when viewing them as a blog post. This suggests that the use of VR technology to promote the hotel did generate a sense of presence for the audience. Past studies have focused on testing the effects of animated VR content, such as Second Life, on audience perception. Although several studies have explored the experience of presence in the virtual depiction of a genuine environment (Tussyadiah et al. 2017, 2018), none have demonstrated the effects of genuine VR content, specifically advertorials in the form of VR $360^{\circ}$ video, on audiences' attitudes and purchase intentions, and compared these effects with content viewed without VR technology. The findings of the present study confirm the persuasiveness of genuine VR content, which is becoming an increasingly prominent tool for consumer marketing and the tourism industry.

This was also one of the first studies to examine the use of Cardboard goggles and VR $360^{\circ}$ video on mobile platforms, which is a recent trend in VR applications in the tourism industry. Past studies found that Cardboard goggles evoked a lower level of presence than high-end wearable devices (Chessa et al. 2016). This study contributes to the literature by showing that presenting a VR $360^{\circ}$ video to an audience wearing Cardboard goggles did not produce a significantly greater sense of presence than showing a VR $360^{\circ}$ video to an audience without goggles. Although Cardboard users are insulated from the outside world by having their eyes covered, placing them at the center of the scene portrayed, there are limitations to viewing $360^{\circ}$ videos through Cardboard goggles. Unlike Cardboard, more robust VR headsets track head movements using sensors, with a computer helping to match the position and orientation of the head with a corresponding view of the virtual world. Cardboard, in contrast, cannot check the head position and lacks fine control over display timing, and so it cannot offer some of the key features that make a virtual environment feel real to an audience. These limitations might explain why viewing a VR $360^{\circ}$ video with Cardboard goggles did not evoke a greater intensity of presence than the use of mobile phones alone in this study. In addition, Cardboard goggles can cause motion sickness and even induce nausea and vomiting if they are worn for too long (Smith, 2015). Future researchers could compare the effects of viewing $360^{\circ}$ video using Cardboard and VR headsets to explore the contributions made by different VR equipment to the experience of presence.

Another key finding is that sense of presence mediated the effects of VR technology on the audience's attitudes toward the product and their purchase intentions. A greater intensity of presence reported by the participants when viewing the 
advertorials using VR technology corresponded to a more favorable attitude toward the advertised product, and thus to a greater purchase intention. Specifically, participants who were more immersed in the advertorials evaluated the advertised hotel as being of a higher quality and were more willing to book a stay at the hotel. These findings confirm the influence of presence on consumption attitudes.

The results support the TIM, according to which VR transports users out of the real world and incorporates them into the virtual environment, making them more susceptible to the ideas promoted in the immersive medium (Lo and Cheng 2015; Cheng and Lo 2015). Consistent with past studies, significant relationships were found between immersive experience, presence, and the persuasiveness of the advertisement (Hoffman and Novak 1996; Li et al. 2001). The findings suggest that the TIM provides a good theoretical basis for future VR research.

There are several implications of the findings for tourism advertisers seeking to enhance the effectiveness of their promotional materials. First, the results indicate that presence is a significant mediator of the attitudes of an audience toward an advertised product and thus their behavioral intention. Promotional content and delivery should thus be developed to maximize the intensity of the audience's experience of presence, which in turn positively influences their consumption response. Creating a sense of presence is a good strategy for enhancing the effectiveness of a marketing campaign. Second, advertisers should consider the several variables that may affect the experience of presence. The results suggest that it is important to enhance the immersion and interactivity for viewers. However, the use of Cardboard goggles was not found to enhance the intensity of viewers' sense of presence beyond that offered by viewing content on a mobile phone without a wearable device. VR technology is constantly evolving, but with budgets limited, especially against the backdrop of the COVID-19 pandemic, advertisers should test carefully before applying new VR innovations. Variables related to content (e.g., voice, background music, shooting angle) and other structural variables (e.g., headset, communication modality) may increase the intensity of the audience's sense of presence when viewing promotional content.

Practical implications aside, there are some limitations to this study. First, as convenience sampling was used to recruit college students, the generalizability of the results to other demographic groups is low. Future researchers should replicate this study with samples from other demographic groups. Second, the stimuli were viewed in university classrooms, which may differ from the real-life environments in which viewers are exposed to advertising content. This may have reduced the ecological validity of the study. Third, only one destination was tested in the study. Future researchers could include other destinations to compare the use of VR technology between travel contexts. Fourth, the interactivity of the VR videos used in the study was quite low. Future researchers could include other interactive features in the content used for experimentation, as interactivity has been found to influence viewers' sense of presence (Fortin and Dholakia 2005). Fifth, the selected stimulus was an actual VR video that had been released to the market, and technical limitations prevented the removal of the logo of the media organization from the video. The participants may thus have been able to identify the organization, which may have influenced their perceptions of the video and the hotel advertised, as evaluation 
of the source of media content has been found to influence evaluation of the content itself (Cheng and Lo 2012).

\section{Conclusions}

This was one of the first studies to examine the effects of using genuine VR content and Google Cardboard goggles on viewers' perceptions. The findings highlight the role of presence in mediating the relationship between an audience's exposure to a VR video and their attitudes and purchase intentions toward the advertised product. The viewers perceived the advertised hotel more favorably and showed a greater intention to book rooms at the hotel when they experienced a more intense sense of presence through the use of VR $360^{\circ}$ video technology (compared with viewing a blog post); however, the use of Cardboard goggles did not enhance their sense of presence relative to the use of a mobile phone alone. There have been huge investments in VR technology recently as it embarks a new phase of development. VR has become an increasingly prominent tool for consumer marketing, as the technology enables advertisers to provide potential consumers with a realistic virtual experience of a product. This has captured the attention of tourism advertisers, but also other segments of the advertising industry, such as motor vehicle marketing. With the recent substantial increase in the use of VR technology for advertising, especially in the tourism industry, media scholars and practitioners should optimize the use of this innovative new technology by paying more attention to the impact of VR technology on the perceptions of audiences, and to the factors that affect their experiences of virtual environments.

\section{References}

Allen DG, Mahto RV, Otondo RF (2007) Web-based recruitment: effects of information, organizational brand, and attitudes toward a web site on applicant attraction. J Appl Psychol 92(6):1696-1708

Ali F (2016) Hotel website quality, perceived flow, customer satisfaction and purchase intention. J Hosp Tour Technol 7(2):213-228

Andrews JC, Akhter SH, Durvasula S, Muehling D (1992) The effects of advertising distinctiveness and message content involvement on cognitive and affective responses to advertising. J Curr Issues Res Advert 14:45-58

Bagozzi RP, Tybout AM, Craig CS, Sternthal B (1979) The construct validity of the tripartite classification of attitudes. J Mark Res 16:88-95

Barnes S (2016) Understanding virtual reality in marketing: Nature, implications and potential. https:// ssrn.com/abstract=2909100. Accessed 26 Jun 2017

Beck J, Rainoldi M, Egger R (2019) Virtual reality in tourism: a state-of-the art review. Tour Rev 74(3):586-612

Bellini H (2016) Profiles in innovation: the real deal with virtual and augmented reality. http:// www.goldmansachs.com/our-thinking/pages/virtual-and-augmented-reality.html?cid =PS_02_04_07_00_01_16_01. Accessed 26 Jun 2017

Beerli A, Santana JDM (1999) Design and validation of an instrument for measuring advertising effectiveness in the printed media. J Curr Issues Res Advert 21(2):11-30

Biocca F, Delaney B (1995) Immersive virtual reality technology. In: Biocca F, Levy MR (eds) Communication in the age of virtual reality. Erlbaum, Hillsdale, pp 57-124 
Bollen KA (1990) Overall fit in covariance structure models: two types of sample size effects. Psychol Bull 107(2):256-259

Bracken CC (2005) Presence and image quality: the case of high-definition television. Media Psychol 7(2):191-205

Cheng BKL, Lo WH (2012) Can news be imaginative? An experiment testing the perceived credibility of melodramatic animated news, news organizations, media use, and media dependency. The effects of melodramatic animation in crime-related news. Electron News 6(3):131-150

Cheng BKL, Lo WH (2015) The effects of melodramatic animation in crime-related news. Journal Mass Commun Q 92(3):559-579

Cheng BKL, Lo WH (2017) Melodramatic animation, presence, and sympathy for crime victims in news: an experiment with adolescents in Hong Kong. Paper presented at Association for Education in Journalism and Mass Communication, Chicago, Illinois

Chessa S, Corradi A, Foschini L, Girolami M (2016) Empowering mobile crowdsensing through social and ad hoc networking. IEEE Commun Mag 54(7):108-114

Cogburn J, Silcox M (2014) Against brain-in-a-vatism: on the value of virtual reality. Philos Technol 27(4):561-579

Cyr D, Hassanein K, Head M, Ivanov A (2007) The role of social presence in establishing loyalty in e-service environments. Interact Comput 19:43-56

Debbabi S, Baile S (2005) Creating telepresence in virtual environments: overview and critical perspectives. In: Dasgupta $S$ (ed) The encyclopedia of virtual communities and technologies. Idea Group Reference, Hershey, pp 73-78

Drenger J, Gaus H, Jahn S (2008) Does flow influence the brand image in event marketing? J Advert Res 48(1):138-147

Eagly AH, Chaiken S (1993) The psychology of attitudes. Harcourt Brace College Publishers, New York

Fishbein M, Ajzen I (1975) Belief, attitude, intention and behavior: an introduction to theory and research. Addison-Wesley, Reading

Fortin DR, Dholakia RR (2005) Interactivity and vividness effects on social presence and involvement with a web-based advertisement. J Bus Res 58:387-396

Fox J, Arena D, Bailenson JN (2009) Virtual reality: a survival guide for the social scientist. J Media Psychol 21(3):95-111

Giner-Sorolla R (1999) Affect in attitude: immediate and deliberative perspectives. In: Chaiken S, Trope Y (eds) Dual-process theories in social psychology. The Guilford Press, New York, pp 441-461

Grabe ME, Matthew L, Reich RD, Bracken Cheryl C, Ditton Theresa B (1999) The role of screen size in viewer experiences of media content. Visual Commun Q 6(2):4-9

Green MC, Brock TC (2000) The role of transportation in the persuasiveness of public narratives. J Pers Soc Psychol 79(5):701-721

Grigorovici D (2003) Persuasive effects of presence in immersive virtual environments. In: Riva G, Davide F, Ijsselstenijn W (eds) Being there: concepts, effects and measurement of presence in synthetic environment. Ios Press, Amsterdam, pp 191-205

Gutierrez M, Vexo F, Thalmann D (2008) Stepping into virtual reality. Springer, London

Guttentag DA (2010) Virtual reality applications and implications for tourism. Tour Manag 31:637-651. https://doi.org/10.1016/j.tourman.2009.07.003

Hamman B (2006) Two voices: social presence, participation, and credibility in online news (Unpublished doctoral dissertation). University of Missouri Columbia

Hoffman DL, Novak TP (1996) Marketing in hypermedia computer-mediated environments: conceptual foundations. J Market 60(3):50-68

Hou J, Nam Y, Peng W, Lee KM (2012) Effects of screen size, viewing angle, and player's immersion tendencies on game experience. Comput Hum Behav 28(2):617-623

Huang MH (2003) Designing website attributes to induce experiential encounters. Comput Hum Behav 19(4):425-442

Huang YC, Backman SJ, Backman KF (2012) Exploring the impacts of involvement and flow experiences in second life on people's travel intentions. J Hosp Tour Technol 3(1):4-23

Huang YC, Backman KF, Backman SJ, Chang LL (2016) Exploring the implication of virtual reality technology in tourism marketing: an integrated research framework. Int J Tour Res 18(2):116-128

Jon B (2016) VR Photojournalism gets real. Photo District News 36(7):40-43

Jung T, Chung N, Leue MC (2015) The determinants of recommendations to use augmented reality technologies: the case of a Korean theme park. Tour Manag 49:75-86 
Kabrovski R (2017) Virtual reality and the future of social media. Digital Mmarketing Mmagazine. http:// digitalmarketingmagazine.co.uk/social-media-marketing/virtual-reality-and-the-future-of-socia 1-media/4745

Kim T, Biocca F (1997) Telepresence via television: two dimensions of telepresence may have different connections to memory and persuasion. J Comput-med Commun. https://doi. org/10.1111/j.1083-6101.1997.tb00073.x

Klein LR (2003) Creating virtual product experiences: the role of telepresence. J Interact Mark 17(1):41-55

Lee KM (2004) Presence, explicated. Commun Theory 14:27-50

Li H, Daugherty T, Biocca F (2001) Characteristics of virtual experience in electronic commerce: a protocol analysis. J Interact Mark 15(3):13-30

Li H, Daugherty T, Biocca F (2002) Impact of 3-D advertising on product knowledge, brand attitude, and purchase intention: the mediating role of presence. J Advert 31(3):43-57. https://doi. org/10.1080/00913367.2002.10673675

Lichterman J (2017) The New York Times' latest VR project is an adaptation of George Saunders' new novel. NiemanLab. https://www.niemanlab.org/2017/02/the-new-york-times-latest-vr-project-is-anadaptation-of-george-saunders-new-novel/

Lo WH, Cheng BKL (2015) The use of melodramatic animation in news, presence and news credibility: a path model. Journal Stud. https://doi.org/10.1080/1461670X.2015.1087814

Limniou M, Roberts D, Papadopoulos N (2008) Full immersive virtual environment CAVETM in chemistry education. Comput Educ 51(2):584-593

Lombard M, Ditton TB (1997) "At the heart of it all: the concept of presence. J Comput-Med Commun 3(2). http://jcmc.indiana.edu/vol3/issue2/lombard.html. Accessed 14 Feb 2018

Lombard M, Ditton TB, Davis CD, Gil-Egui B, Horvath G, Rossman KJ (2000) Measuring presence: a literature-based approach to the development of a standardised paper-and-pencil instrument. The third international workshop. https://astro.temple.edu/ lombard/ISPR/Proceedings/2000/Lomba rd,\%20Ditton\%20et\%20al.pdf. Accessed 14 Feb 2018

Mascho E, Singh N (2013) Virtual tourism: use of "second life" for destination marketing. Anatolia 25(1):140-143. https://doi.org/10.1080/13032917.2013.815644

Mandalbaum A (2015) How companies are marketing with virtual reality. http://www.chiefmarketer.com/ companies-marketing-virtual-reality/. Accessed 26 Jun 2017

Mitchell AA, Olson JC (1981) Are product beliefs the only mediator of advertising effect on brand attitude? J Mark Res 18(August):318-332

McDowell W, Sutherland J (2000) Choice versus chance: using brand equity theory to explore TV audience lead-in effects, a case study. J Media Econ 13(4):233-247

Molla R (2017) These are the companies investing most aggressively in AR and VR: Facebook, HTC and Intel, among others. https://www.recode.net/2017/4/28/15376268/facebook-augmented-virtual-reali ty-linkedin-jobs-charts

Moorthy S, Hawkins SA (2005) Advertising repetition and quality perception. J Bus Res 58(3):354-360

Morris C (2016) Virtual reality and the new sales experience. http://www.campaignlive.co.uk/article/ virtual-reality-new-sales-experience/1392253. Accessed 27 Jun 2017

Morris JD, Woo C, Geason JA, Kim J (2002) The power of affect: predicting intention. J Advert Res 42(3):7-17

Neuman WR (1990) Beyond HDTV: exploring subjective responses to very high definition television. Research report for GTE Labs and the TVOT consortium. MIT Press, Cambridge

Nowak KL, Biocca F (2003) The effect of the agency and anthropomorphism on Users' sense of telepresence. Presence Teleoper Virtual Environ 12(5):481-494

Park S, Nah F, DeWester D, Eschenbrenner B, Jeon S (2008) Virtual world affordances: enhancing brand value. J Virtual Worlds Res 1(2):1-18

Penfold P (2009) Learning through the world of second life-a hospitality and tourism experience. J Teach Travel Tour 8(2-3):139-160. https://doi.org/10.1080/15313220802634224

Reeves B, Nass C (1996) The media equation: how people treat computers, television, and sources relate to beliefs about media bias. Newsp Res J 20(4):41-51

Reid D (2004) A model of playfulness and flow in virtual reality interactions. Presence Teleoper Virtual Environ 13(4):451-462

Rizzo AA, Wiederhold M, Buckwalter JG (1998) Basic issues in the use of virtual environments for mental health applications. In: Riva G, Wiederhold B, Molinari E (eds) Virtual environments in clinical psychology and neuroscience. IOS Press, Amsterdam, pp 22-24 
Sääksjärvia M, Samiee S (2011) Relationships among brand identity, brand image and brand preference: differences between cyber and extension retail brands over time. J Interact Mark 25(3):165-177

Sharples S, Cobb S, Moody A, Wilson JR (2008) Virtual reality induced symptoms and effects (VRISE): comparison of head mounted display (HMD), desktop and projection display systems. Displays 29(2):58-69

Shinta VR (2017) Google venture's investment strategy for virtual reality. http://shintavr.com/en/news/ index/105/Google-Venture---s-Investment-Strategy-for-Virtual-Reality. Accessed 10 Sept 2020

Simone LK, Schultheis MT, Rebimbas J, Millis SR (2006) Head-mounted displays for clinical virtual reality applications: pitfalls in understanding user behavior while using technology. CyberPsychol Behav 9(5):591-602

Skyscanner (2017) Top 10 most google-searched tourist destinations among Hong Kong people. https ://www.skyscanner.com.hk/news/10-most-google-searched-destinations-of-hong-kongers-201701 (Chinese website). Accessed 10 Sept 2020

Smith W (2015) Stop calling google Cardboard's 360-degree videos VR. Wired. https://www.wired .com/2015/11/360-video-isnt-virtual-reality/. Accessed 10 Sept 2020

Spear N, Singh SN (2004) Measuring attitude toward the brand and purchase intentions. J Curr Issues Res Advert 26(2):53-66

Steuer J (1992) Defining Virtual reality: dimensions determining telepresence. J Commun 42(4):73-93

Strange JJ, Leung CC (1999) How anecdotal accounts in news and in fiction can influence judgements of a social problem's urgency, causes, and cures. Pers Soc Psychol Bull 25:436-449

Sylaiou S, Mania K, Karoulis A, White M (2010) Exploring the relationship between presence and enjoyment in a virtual museum. Int J Hum-Comput Stud 68(5):243-253

Tavakoli R, Mura P (2015) Journeys in Second Life'-Iranian Muslim women's behavior in virtual tourist destinations. Tour Manag 46:398-407

The Guardian (2017) The Guardian and VR. Here are the answers to all the questions you might ask. https:/www.theguardian.com/technology/ng-interactive/2016/nov/10/virtual-reality-by-the-guard ian

Tussyadiah IP, Wang D, Jia CH (2017) Virtual reality and attitudes toward tourism destinations. In: Schegg R, Stangl B (eds) Information and communication technologies in tourism 2017. Springer, Cham, pp 229-239

Tussyadiah IP, Wang D, Jung TH, Tom Dieck MC (2018) Virtual reality, presence, and attitude change: Empirical evidence from tourism. Tour Manag 66:140-154

Villani D, Riva F, Riva G (2007) New technologies for relaxation: the role of presence. Int J Stress Manag $14: 260-274$

Watson Z (2017) VR for news: the new reality? Digital News Publications. http://www.digitalnewsrepo rt.org/publications/2017/vr-news-new-reality/. Accessed 10 Sept 2020

William P, Hobson JP (1995) Virtual reality: facts of fantasy? Tour Manag 16(6):423-427

Witmer BG, Jerome CJ, Singer MJ (2005) The factor structure of the presence questionnaire. Presence Teleoper Virtual Environ 14:298-312

Wreford O, Williams NL, Ferdinand N (2019) Together alone: an exploration of the virtual event experience. Event Manag 23(4-5):721-732. https://doi.org/10.3727/152599519X15506259855625

Wu D-Y, Lin J-HT (2018) Ways of seeing matter: the impact of a naturally mapped perceptual system on the persuasive effects of immersive virtual reality advertising. Commun Res Rep 35(5):434-444. https://doi.org/10.1080/08824096.2018.1525349

Yoon Y, Uysal M (2005) An examination of the effects of motivation and satisfaction on destination loyalty: a structural model. Tour Manag 26:45-56

YouGov (2017) VR headsets more popular than tablets and wearables were at same stage. https://yougo v.co.uk/news/2017/05/19/vr-headsets-more-popular-tablets-and-wearables-wer/. Accessed 10 Sept 2020

Yung R, Khoo-Lattimore C (2019) New realities: a systematic literature review on virtual reality and augmented reality in tourism research. Curr Issues Tour 22(17):2056-2081. https://doi. org/10.1080/13683500.2017.1417359

Zaragoza MS, Mitchell KJ (1996) Repeated exposure to suggestion and the creation of false memories. Psychol Sci 7(5):294-300

Publisher's Note Springer Nature remains neutral with regard to jurisdictional claims in published maps and institutional affiliations. 